Pesq. Vet. Bras. 29(3):275-280, março 2009

Tópico de Interesse Geral

\title{
Achados de necropsia relacionados com a morte de 335 eqüinos: $1968-2007^{1}$
}

\author{
Felipe Pierezan², Daniel R. Rissi², Raquel R. Rech ${ }^{3}$, Rafael A. Fighera ${ }^{4}$, \\ Juliana S. Brum ${ }^{5}$ e Claudio S.L. Barros ${ }^{4 *}$
}

\begin{abstract}
Pierezan F., Rissi D.R., Rech R.R., Fighera R.A. Brum J.S. \& Barros C.S.L. 2009. [Necropsy findings related to the cause of death in 335 horses: 1968-2007.] Achados de necropsia relacionados com a morte de 335 eqüinos: 1968-2007. Pesquisa Veterinária Brasileira 29(3):275-280. Departamento de Patologia, Universidade Federal de Santa Maria, 97105-900 Santa Maria, RS, Brazil. E-mail: claudioslbarros@uol.com.br

The necropsy reports of 335 horses necropsied at the LPV-UFSM between 19682007 were reviewed in order to determine the necropsy findings related with cause of death or reason for euthanasia. The distribution of these findings by organ system were as follows: digestive (79/335 [23.6\%]), striated muscle and skeleton (47/335 [14.0\%]), nervous (37/335 [11.0\%]), respiratory (35/335 [10.4\%]), integument (31/335 [9.3\%]), hematopoietic $(24 / 335[7.2 \%])$, cardiovascular $(13 / 335$ [3.9\%]), reproductive $(12 / 335$ $[3.5 \%])$, urinary $(7 / 335[2.1 \%])$, and endocrine $(3 / 335[0.9 \%])$. The cause of death was not possible to be determined in $47(14.0 \%)$ necropsied horses. Displacements of the intestines (17/79 [21.5\%]) were the main findings in digestive system, followed by obstruction and impactation (14/79 [17.7\%]). Torsion were the type of displacement more frequently observed in the intestines (14/17 [82.4\%]). Among those the more prevalent affected the small intestine (7/14 [50\%]). Most horses dying from fractured bones were 15 -year-old. The most prevalent diseases in the nervous system were leukoencephalomalacia and trypanosomiasis, whereas respiratory depression due to anesthesia was the leading cause of death related to the respiratory system. Equine infectious anemia was the most diagnosed infectious disease and the main reason leading to euthanasia.
\end{abstract}

INDEX TERMS: Diseases of horses, causes of death, epidemiology, pathology.

RESUMO.- Os protocolos de necropsia de 335 eqüinos necropsiados no LPV-UFSM entre 1968-2007 foram revisados com o objetivo de determinar os achados de necropsia relacionados com a causa da morte ou razão de

\footnotetext{
${ }^{1}$ Recebido em 12 se novembro de 2008.

Aceito para publicação em 4 de dezembro de 2008.

Parte da Dissertação de Mestrado do primeiro autor.

2 Programa de Pós-graduação em Medicina Veterinária, área de concentração em Patologia Veterinária, Centro de Ciências Rurais, Universidade Federal de Santa Maria (UFSM).

${ }^{3}$ Department of Veterinary Pathology, College of Veterinary Medicine, University of Georgia, Athens, GA 30602-7388, Estados Unidos.

${ }^{4}$ Departamento de Patologia, UFSM, 97105-900 Santa Maria, Rio Grande do Sul. *Pesquisador $1 \mathrm{~A}$ do CNPq. Autor para correspondência claudioslbarros@uol.com.br

${ }^{5}$ Bolsista de Iniciação científica do CNPq junto ao Departamento de Patologia da UFSM.
}

eutanásia. A distribuição desses achados de acordo com os sistemas afetados foi a seguinte: digestivo (79/335 $[23,6 \%])$, músculo-esquelético $(47 / 335$ ([14,0\%]), nervoso $(37 / 335[11,0 \%])$, respiratório $(35 / 335[10,4 \%])$, tegumentar (31/335 [9,3\%]), hematopoético (24/335 [7,2\%]), cardiovascular (13/335[3,9\%]), reprodutor (12/335 [3,5\%]), urinário $(7 / 335[2,1 \%])$ e endócrino $(3 / 335[0,9 \%])$. Não foi possível determinar a causa da morte em 47 (14,0\%) eqüinos necropsiados. As principais afecções do sistema digestivo foram as alterações na posição dos intestinos (17/79 [21,5\%]), seguidas pelas obstruções e impactações (14/79 [17,7\%]). As torções foram as principais alterações da posição dos intestinos (14/17 [82,4\%]). Dentre as torções, as mais prevalentes foram as localizadas no intestino delgado (7/14 [50\%]). A maioria dos eqüinos que morreram em razão de fratura óssea tinham idades entre 
1-5 anos. As duas doenças mais freqüentemente diagnosticadas no sistema nervoso foram leucoencefalomalacia e tripanossomíase. Depressão respiratória causada por anestesia foi a principal causa de morte relacionada com o sistema respiratório. A anemia infecciosa eqüina foi a doença infecciosa mais diagnosticada e a principal razão para eutanásia observada neste estudo.

TERMOS DE INDEXAÇÃO: doenças de eqüinos, causas de morte, epidemiologia, patologia.

\section{INTRODUÇÃO}

Estudos retrospectivos sobre causas de morte ou razões para eutanásia procuram determinar a prevalência de doenças em relação à espécie, ao sexo, à raça e à idade em uma determinada região. Adicionalmente, são ferramentas utilizadas para agrupar dados clinicopatológicos e laboratoriais sobre enfermidades, auxiliando clínicos e patologistas em sua rotina diagnóstica. A partir desses estudos é possível desenvolver métodos de prevenção e controle relacionados a características específicas de uma determinada doença, como, por exemplo, época de ocorrência ou faixa etária dos animais afetados (Martins 1987).

Há poucos estudos sobre a prevalência de doenças de eqüinos no Brasil e esses estudos são restritos a sistemas ou órgãos específicos (Pimentel et al. 2007, Reichmann et al. 2008, Trotte et al. 2008). O objetivo deste estudo foi analisar os protocolos de necropsias de eqüinos realizadas no Laboratório de Patologia Veterinária (LPV) da Universidade Federal de Santa Maria (UFSM) de 1968-2007, a fim de estabelecer as causas de morte e razões para eutanásia nessa espécie e relacionar os achados com o sexo, a raça e a idade da população estudada.

\section{MATERIAL E MÉTODOS}

Foram analisados os dados referentes ao sexo, raça e idade e achados clinicopatológicos dos 335 eqüinos necropsiados no LPV-UFSM entre 1968 e 2007. Quanto ao sexo, os eqüinos foram divididos em machos, machos castrados e fêmeas. Quanto à idade, os eqüinos foram divididos nas seguintes categorias: menores de 1 ano, 1-5 anos, 6-14 anos e 15 anos ou mais. As categorias utilizadas foram adaptadas da divisão utilizada em um estudo sobre causas de morte de eqüinos realizado na Suécia (Egenvall et al. 2005) e justificam-se pelas diferenças entre as doenças que afetam cada uma dessas faixas etárias. As causas de morte e as razões para eutanásia foram agrupadas de acordo com os sistemas afetados. A análise estatística da associação entre determinadas afecções e a faixa etária de ocorrência foi feita pelo teste do Qui-quadrado (X2), com intervalo de confiança determinado por $p$ menor ou igual a 0,05, utilizando-se o programa estatístico SAS (Sas 1990). Em determinadas afecções, devido ao número reduzido de casos, não foi possível aplicar um método estatístico capaz de determinar a significância dos dados obtidos.

\section{RESULTADOS E DISCUSSÃO}

A distribuição do número de necropsias por décadas foi a seguinte: 58 (17,3\%) casos de 1968-1977, 69 (20,6\%) de
1978-1987, 107 (31,9\%) de 1988-1997 e 101 (30,1\%) de 1998-2007. Do total de eqüinos que tiveram o sexo informado nos protocolos de necropsia, $159(47,5 \%)$ eram machos, 20 (6,0\%) eram machos castrados e $148(44,2 \%)$ eram fêmeas. Em oito casos (2,4\%) o sexo não foi informado.

Em 66 (19,7\%) protocolos de necropsia a idade não foi informada. Dos 269 eqüinos que tiveram a idade informada, $46(13,7 \%)$ tinham até um ano de idade, 102 $(30,4 \%)$ tinham $1-5$ anos, 93 (27,7\%) tinham 6-14 anos e $28(8,3 \%)$ tinham 15 anos ou mais. A idade dos eqüinos variou de um dia a 40 anos (média de 6,5 anos).

Em $76(22,7 \%)$ casos não havia informação sobre a raça. Dos 259 eqüinos que tiveram esse parâmetro informado nos protocolos de necropsia, havia $101(39,0 \%)$ eqüinos sem raça definida e $158(61,0 \%)$ eqüinos de raça pura, que incluíam: Puro Sangue de Corrida (96/259 [37,0\%]), Crioulo (46/259 [17,8\%]), Quarto-de-Milha (9/259 $[3,5 \%])$, Hannoverano (5/259 [1,9\%]), Árabe (1/259 [0,4\%]) e Brasileiro de Hipismo (1/259 [0,4\%]).

A distribuição das doenças por sistemas pode ser observada nos Quadros 1-4. O sistema mais afetado foi o digestivo (79/335 [23,6\%]), seguido pelo músculoesquelético (47/335 [14,0\%]), nervoso (37/335 [11,0\%]), respiratório (35/335 [10,4\%]), tegumentar (31/335 [9,3\%]), hematopoético (24/335 [7,2\%]), cardiovascular (13/335 $[3,9 \%])$, reprodutor $(12 / 335[3,5 \%])$, urinário $(7 / 335[2,1 \%])$ e endócrino (3/335 [0,9\%]). Dos 335 casos, $47(14,0 \%)$ não tinham um diagnóstico conclusivo.

Resultados semelhantes quanto à distribuição das afecções por sistema foram observados por outros autores. Num estudo retrospectivo sobre causas de morte de eqüinos na Inglaterra (Baker \& Ellis 1981), os sistemas mais afetados foram o digestivo $(33,1 \%)$, seguido pelo músculo-esquelético $(15,8 \%)$, nervoso $(15,8 \%)$, respiratório $(8,5 \%)$, cardiovascular $(8,5 \%)$, hematopoético $(4,5 \%)$, urinário $(3,3 \%)$, tegumentar $(1,6 \%)$ e $3,1 \%$ de casos inconclusivos. No Brasil, há um levantamento sobre causas de morte de eqüinos na Paraíba (Pimentel 2007); em 98 protocolos de necropsia analisados, os sistemas mais afetados foram nervoso $(26,5 \%)$, digestivo $(21,4 \%)$, músculo-esquelético $(21,4 \%)$, respiratório $(7,1 \%)$, tegumen$\operatorname{tar}(5,1 \%)$, hematopoético (1\%) e cardiovascular $(1 \%)$. O índice de casos inconclusivos $(16,3 \%)$ foi muito semeIhante ao observado neste estudo (14,0\%). Embora exista semelhança na distribuição das afecções por sistemas, a prevalência de determinadas doenças difere muito entre os estudos analisados. Por exemplo, no estudo inglês (Baker \& Ellis 1981), as doenças mais prevalentes dos sistemas digestivo, musculo-esquelético e nervoso foram, respectivamente, as perfurações, as fraturas e a disautonomia, que foi a doença individual mais prevalente do estudo. No estudo brasileiro (Pimentel et al. 2007), a principal afecção do sistema nervoso central e a segunda mais prevalente do levantamento, superada apenas pelas fraturas, foi a encefalopatia hepática secundária a cirrose hepática associada à intoxicação crônica por Crotalaria 
Quadro 1. Causas de morte em eqüinos. Afecções do sistema digestivo $(n=79)$ encontradas em 335 necropsias

\begin{tabular}{|c|c|}
\hline Diagnóstico & Número de casos \\
\hline Alterações da posição dos intestinos & 17 \\
\hline Torção intestinal & 14 \\
\hline Intestino delgado & 7 \\
\hline Cólon maior & 4 \\
\hline Ceco & 2 \\
\hline Cólon menor & 1 \\
\hline Deslocamento do cólon maior & 1 \\
\hline Hérnia umbilical & 1 \\
\hline Encarceramento de alça intestinal & 1 \\
\hline Obstruções e impactações intestinais & 14 \\
\hline Corpos estranhos & 9 \\
\hline Cólon menor & 6 \\
\hline Cólon maior & 2 \\
\hline Ceco & 1 \\
\hline Impactação de cólon maior & 2 \\
\hline Abscesso no intestino delgado & 1 \\
\hline Aderências no intestino delgado & 1 \\
\hline Intussuscepção de intestino delgado & 1 \\
\hline Inflamações & 13 \\
\hline Enterites e colites inespecíficas & 7 \\
\hline Enterite por ciatostomíneos & 5 \\
\hline Colite X & 1 \\
\hline Dilatações gástricas & 5 \\
\hline Dilatações gástricas sem ruptura & 4 \\
\hline Dilatações gástricas com ruptura & 1 \\
\hline Peritonite & 3 \\
\hline Peritonite secundária à perfuração abdominal & 2 \\
\hline Peritonite sem causa definida & 1 \\
\hline Perfurações por corpos estranhos & 2 \\
\hline Faringe & 1 \\
\hline Intestino delgado & 1 \\
\hline Úlceras gástricas & 2 \\
\hline Necrose hepática tóxica & 2 \\
\hline Cirrose hepática (intoxicação por Senecio sp.) & 1 \\
\hline Linfoma intestinal & 1 \\
\hline Timpanismo de cólon maior & 1 \\
\hline Condições iatrogênicas & 12 \\
\hline Eventração de alças intestinais & 3 \\
\hline Ruptura traumática de cólon menor & 2 \\
\hline Esofagite necrosante & 2 \\
\hline Peritonite por contaminação de ferida cirúrgica & 2 \\
\hline Traumatismo de faringe & 2 \\
\hline Ruptura de incisão cirúrgica no flanco esquerdo & 1 \\
\hline Caquexia & 4 \\
\hline Inanição & 2 \\
\hline
\end{tabular}

retusa, o que determinou a alta prevalência de lesões desse sistema em eqüinos daquele estudo.

Neste estudo, as doenças do sistema digestivo foram as mais prevalentes. Os eqüinos apresentam peculiaridades anatômicas no sistema digestivo que podem predispor essa espécie a alterações morfológicas graves. Exemplos dessas particularidades incluem (1) a baixa capacidade do estômago em relação à capacidade digestiva total; (2) a longa extensão do intestino delgado que é fixado apenas por um mesentério muito desenvolvido; (3) a mobilidade do cólon maior e do ceco, que são presos apenas pela raiz mesentérica; (4) a existência de locais de diminuição da luz intestinal como a flexura pélvica; e (5) a incapacidade de eructar e regurgitar (Godoy \& Neto 2007). Essas particularidades podem explicar a alta pre-
Quadro 2. Causas de morte em eqüinos. Afecções do sistema músculo-esquelético $(n=47)$ encontradas em 335 necropsias

\begin{tabular}{lc}
\hline \multicolumn{1}{c}{ Diagnóstico } & Número de casos \\
\hline Fraturas fisiológicas & 20 \\
Membro torácico & 12 \\
Membro pélvico & 5 \\
Vértebras & 2 \\
Mandíbula & 1 \\
Fraturas patológicas (osteodistrofia fibrosa) & 4 \\
Vértebras & 3 \\
Fêmur & 1 \\
Necrose muscular & 9 \\
Necrose muscular por traumatismo & 3 \\
Necrose muscular por intoxicação por monensina & 2 \\
Necrose muscular pós-anestésica & 1 \\
Necrose muscular por decúbito & 1 \\
Necrose muscular pós-exercício & 1 \\
Necrose muscular por acidente botrópico & 1 \\
Sinovite & 4 \\
Laminite e pododermatite & 3 \\
Artrite e osteomielite & 2 \\
Ruptura de tendão ou ligamento & 2 \\
Luxação vertebral & 1 \\
Miosite necrossupurativa & 1 \\
Hemangiossarcoma vertebral & 1
\end{tabular}

valência de afecções do sistema digestivo neste e em outros estudos sobre causas de mortes em eqüinos (Baker \& Ellis 1981, Pimentel et al. 2007).

As principais afecções do sistema digestivo foram as alterações na posição dos intestinos (17/79 [21,5\%]), seguidas pelas obstruções e impactações (14/79 [17,7\%]). As torções foram as principais alterações da posição dos intestinos (14/17 [82,4\%]). As mais prevalentes foram no intestino delgado (7/14 [50\%]). Algumas formas de alterações da posição dos intestinos, que cursam clinicamente com cólica, parecem ser mais prevalentes em animais jovens, como as intussuscepções (Gift et al. 1993) ou hérnias umbilicais (Fretz et al. 1983), enquanto outras são mais prevalentes em eqüinos adultos e idosos, como estrangulamento de alça intestinal por lipoma pedunculado (Blikslager et al. 1992) e deslocamentos de cólon maior (Hackett 2002). No entanto neste estudo, não foi observada predisposição por sexo, raça ou idade nos casos de alterações da posição os intestinos.

As principais causas de obstrução intestinal foram os enterólitos (quatro casos), seguidos pelos emaranhados de cordas mineralizados (dois casos), piloconcrementos (dois casos) e sacos plásticos (um caso). Em todos os casos de enterolitíase, em que a idade foi informada, os eqüinos tinham mais de três anos de idade. Em algumas regiões, como no sudeste dos Estados Unidos, observa-se uma alta prevalência de casos de enterolitíase, devido, provavelmente, aos altos níveis de magnésio, fósforo e amônia na dieta. Enterólitos ocorrem predominantemente em eqüinos com mais de quatro anos de idade, enquanto obstruções por corpos estranhos, como sacos plásticos, são mais comuns em eqüinos com menos de três anos de idade, devido ao hábito alimentar menos seletivo nessa faixa etária 
Quadro 3. Causas de morte em eqüinos. Afecções do sistema nervoso $(n=37)$, do sistema respiratório $(n=35)$ e do sistema tegumentar $(n=31)$ encontradas em 335 necropsias

\begin{tabular}{|c|c|}
\hline Diagnósticos & Número de \\
\hline \multicolumn{2}{|l|}{ Sistema nervoso } \\
\hline Meningoencefalite não-supurativa & 9 \\
\hline Leucoencefalomalacia & 8 \\
\hline Tripanossomíase & 7 \\
\hline Mielomalacia segmentar traumática & 4 \\
\hline Tétano & 4 \\
\hline Inflamação/degeneração de nervos periféricos & 3 \\
\hline Harpejamento & 2 \\
\hline Causa não determinada & 1 \\
\hline Encefalomalacia e hemorragia subdural traumátice & ica \\
\hline Mieloencefalite por protozoário & 1 \\
\hline \multicolumn{2}{|l|}{ Sistema respiratório } \\
\hline \multicolumn{2}{|l|}{ Depressão anestésica } \\
\hline \multicolumn{2}{|l|}{ Broncopneumonia } \\
\hline \multirow{2}{*}{\multicolumn{2}{|c|}{ Broncopneumonia por Rhodococcus equi }} \\
\hline & 3 \\
\hline \multicolumn{2}{|l|}{ Pleuropneumonia } \\
\hline Pleuropneumonia aspirativa & 3 \\
\hline Pleuropneumonia de causa não determinada & 2 \\
\hline \multicolumn{2}{|l|}{ Pneumonia intersticial } \\
\hline \multicolumn{2}{|l|}{ Hemotórax } \\
\hline \multicolumn{2}{|l|}{ Piotórax } \\
\hline \multicolumn{2}{|l|}{ Abscesso torácico } \\
\hline Guturocistite purulenta & 1 \\
\hline \multicolumn{2}{|l|}{ Sistema tegumentar } \\
\hline \multicolumn{2}{|l|}{ Neoplasma ou lesões tumoriformes } \\
\hline Pitiose & 12 \\
\hline Carcinoma de células escamosas & 3 \\
\hline Melanoma maligno multicêntrico & 2 \\
\hline Carcinoma indiferenciado & 1 \\
\hline Fibroma & 1 \\
\hline Linfoma & 1 \\
\hline Hamartoma vascular & 1 \\
\hline Sarcóide & 1 \\
\hline Feridas extensas & 6 \\
\hline Abscessos da região da cernelha (macaco) & 2 \\
\hline Sarna sarcóptica & 1 \\
\hline
\end{tabular}

(Schumacher 2002). Perfurações do tubo digestivo e da parede abdominal, com conseqüente peritonite, foram observadas em eqüinos de todas as idades e foram causados por fragmentos de madeira e arames.

A grande maioria dos casos de colite ou enterite observada neste estudo foi classificada como inespecífica devido à ausência de provas diagnósticas para determinação da etiologia dessas lesões. Muitos desses casos possivelmente estavam associados a agentes bacterianos. As colites e enterites inespecíficas e as enterites causadas por parasitas (ciatostomíneos) foram mais freqüentes em eqüinos com menos de um ano de idade $(46,6 \%$ dos casos de alterações inflamatórias) e com 1-5 anos de idade (15,3\% dos casos de alterações inflamatórias), porém, essa diferença não foi significativa. Todos os eqüinos com caquexia tinham mais de 12 anos e foram submetidos à eutanásia devido ao péssimo estado corporal. Os eqüinos com inanição eram neonatos que não ingeriram o colostro e morreram alguns dias após o nascimento.

As afecções mais freqüentes do sistema músculoesquelético foram as fraturas, que corresponderam a apro-
Quadro 4. Causas de morte em eqüinos. Afecções dos

sistemas hematopoético $(n=24)$, cardiovascular (13), reprodutor $(n=12)$, urinário $(n=7)$ e endócrino $(n=3)$ encontradas em 335 necropsias

\begin{tabular}{|c|c|}
\hline Diagnósticos & o de casos \\
\hline \multicolumn{2}{|l|}{ Sistema hematopoético } \\
\hline Anemia hemolítica & 21 \\
\hline Anemia infecciosa eqüina & 14 \\
\hline Isoeritrólise neonatal & 2 \\
\hline Babesiose & 1 \\
\hline Anemia hemolítica de causa não determinada & 4 \\
\hline Abscesso no baço secundário à trombose & 1 \\
\hline Linfadenite purulenta (Streptococcus zooepidemicus) & 1 \\
\hline $\begin{array}{l}\text { Ruptura de baço associada a parasitismo por Strongylus } \\
\text { vulgaris }\end{array}$ & 1 \\
\hline \multicolumn{2}{|l|}{ Sistema cardiovascular } \\
\hline Infartos associados à trombose (Strongylus vulgaris) & 6 \\
\hline Cólon maior & 4 \\
\hline Ceco & 2 \\
\hline Endocardite valvar & 2 \\
\hline Choque endotóxico puerperal & 1 \\
\hline Fibrose e mineralização do miocárdio & 1 \\
\hline Angioleiomiossarcoma da cavidade abdominal & 1 \\
\hline Ruptura de aneurisma da artéria cólica & 1 \\
\hline Ruptura de aorta com tamponamento cardíaco & 1 \\
\hline \multicolumn{2}{|l|}{ Sistema reprodutor } \\
\hline Endometrite & 5 \\
\hline Neoplasmas e lesões tumoriformes & 2 \\
\hline Hamartoma vascular de ovário & 1 \\
\hline Leiomioma uterino & 1 \\
\hline Distúrbios iatrogênicos & 2 \\
\hline Hemorragia de ovário pós-cirúrgica & 2 \\
\hline Ruptura de útero & 2 \\
\hline Fraqueza perinatal (intoxicação por Claviceps purpurea) & 1 \\
\hline \multicolumn{2}{|l|}{ Sistema urinário } \\
\hline Necrose cortical renal & 2 \\
\hline Ruptura de bexiga & 2 \\
\hline Hemorragia renal subcapsular traumática & 1 \\
\hline Prolapso de bexiga & 1 \\
\hline Hamartoma vascular de bexiga & 1 \\
\hline \multicolumn{2}{|l|}{ Sistema endócrino } \\
\hline Bócio (intoxicação por iodo) & 3 \\
\hline
\end{tabular}

ximadamente metade dos casos incluídos nesse sistema. Os principais locais de fraturas fisiológicas foram os membros torácicos (12/20 [60\%]), seguido dos membros pélvicos (cinco casos), vértebras (dois casos) e mandíbula (um caso). Dentre as fraturas patológicas, secundárias à osteodistrofia fibrosa, as vértebras foram os ossos mais afetados (três casos). No outro caso, o local afetado foi o fêmur.

As fraturas ocorreram principalmente em eqüinos com 1-5 anos de idade ( $47,4 \%$ dos casos de fratura), porém não houve diferença significativa em relação à ocorrência dessas afecções nas diferentes faixas etárias. Dados semelhantes quanto à idade dos eqüinos com fraturas foram observados por outros autores (Baker \& Ellis 1981), que verificaram uma maior prevalência de fraturas em eqüinos jovens, com até cinco anos de idade. Provavelmente esse dado está relacionado com a menor resistência dos ossos, com o não fechamento das placas epifisiárias antes dos dois anos de idade e com o início precoce de sua utilização em trabalho ou performance 
(Baker \& Ellis 1981). A maioria dos eqüinos com fraturas foi submetida à eutanásia (19/24 [79,2\% dos casos de fratura]), enquanto apenas dois eqüinos fraturados $(8,3 \%$ dos casos de fratura) morreram naturalmente. Em três casos $(12,5 \%$ dos casos de fratura) de fraturas, a forma da morte não foi informada. A eutanásia de eqüinos fraturados justifica-se pela dificuldade na correção cirúrgica das fraturas.

A leucoencefalomalacia foi a doença mais prevalente no sistema nervoso, perfazendo aproximadamente $20 \%$ dos casos neurológicos. A prevalência dessa condição deve-se a fatores climáticos da região Sul do Brasil, onde a doença tem ocorrência sazonal, principalmente nos meses de junho a setembro, quando há aumento da umidade do ar (Barros et al. 1984, Riet-Correa et al. 1998). Neste estudo, dos oito casos de leucoencefalomalacia diagnosticados no LPV-UFSM, cinco $(62,5 \%)$ casos ocorreram entre os meses de junho-setembro. A idade dos eqüinos com leucoencefalomalacia variou de três meses a 15 anos (média de 6,8 anos). Não houve predisposição por sexo, raça ou idade nesses casos. A tripanossomíase foi a segunda doença mais prevalente do sistema nervoso (7/37 [18,9\% das doenças do sistema nervoso]). As necropsias de eqüinos com tripanossomíase por Trypanossoma evansi faziam parte de surtos ocorridos entre 2003-2004, que causaram a morte de pelo menos 100 eqüinos. Até 2003 essa doença não havia sido diagnosticada no Rio Grande do Sul (Rodrigues et al. 2005, Rodrigues et al. 2009).

Casos de meningoencefalite não-supurativa de causa indeterminada foram bastante prevalentes neste estudo (9/37 [24,3\% das doenças do sistema nervoso]). Em sete casos de meningoencefalite não-supurativa foram realizados testes imuno-histoquímicos para raiva, porém, nenhum resultou positivo. Em três casos foram realizados testes imuno-histoquímicos para febre do Nilo Ocidental e encefalomielites eqüinas tipo leste, oeste e venezuelana, que também foram negativos. É possível que esses resultados negativos possam estar relacionados ao tempo de fixação em formol e à permanência dos tecidos em blocos de parafina, o que pode prejudicar substancialmente esse teste resultando em falso-negativos.

A principal causa de morte relacionada com o sistema respiratório foi a depressão anestésica. A anestesia geral em eqüinos submetidos a procedimentos emergenciais tem um risco maior do que em eqüinos submetidos a cirurgias eletivas. Conforme demonstrado por alguns autores (Mee et al. 1998), a letalidade de eqüinos com cólica submetidos à anestesia geral é aproximadamente 10 vezes maior do que a de eqüinos submetidos a cirurgias eletivas. As broncopneumonias foram a segunda afecção mais prevalente neste sistema. Em dois casos de broncopneumonia foi possível isolar Rhodococcus equi das lesões; no outro caso o diagnóstico baseou-se na epidemiologia e nos achados anatomopatológicos.

A maioria dos eqüinos com afecções do sistema tegumentar foi submetida à eutanásia por opção dos pro- prietários devido à gravidade das lesões ou porque o tratamento é difícil ou oneroso. Dessa forma, 26 eqüinos ( $81,3 \%$ dos casos do sistema tegumentar) foram submetidos à eutanásia, enquanto apenas um eqüino $(3,1 \%)$ morreu naturalmente. Nos demais cinco casos (15,6\% dos casos do sistema tegumentar), a forma da morte do animal não foi informada. Lesões tumoriformes associadas a Pythium insidiosum foram as afecções mais freqüentes no sistema tegumentar. Nos casos de pitiose, não houve predisposição por sexo, raça ou idade. Os principais locais de ocorrência dessas lesões foram o membro pélvico (quatro casos), abdômen ventral (três casos), mandíbula (dois casos), face, região cervical e tórax ventral (um caso cada). Lesões de pitiose são observadas, principalmente, nas porções distais dos membros, principalmente pélvicos, e na região ventral do abdômen. Essas áreas são mais afetadas por serem mais susceptíveis a lesões traumáticas e por permanecerem em maior contato com a água (Sallis et al. 2003). A segunda afecção mais freqüente desse sistema foram os neoplasmas. Houve pouca variação na prevalência dos diferentes neoplasmas cutâneos observados neste estudo.

Os neoplasmas de pele mais comuns em eqüinos incluem o sarcóide, o fibroma, o papiloma, o carcinoma de células escamosas e o melanoma, e a prevalência de determinados neoplasmas em uma região geográfica está associada a fatores climáticos, raças e coloração da pelagem (Valentine 2006).

A anemia infecciosa eqüina (AIE) foi a principal doença infecciosa observada neste estudo, entretanto, os últimos casos de anemia infecciosa eqüina no LPV-UFSM foram diagnosticados em 1977. Apesar disso, essa é uma doença importante e que deve ser mantida sob vigilância e métodos de controle e prevenção devem ser realizados constantemente. Houve maior prevalência de casos de AIE em machos inteiros (12/14 [85,7\%]). As demais anemias hemolíticas (isoeritrólise neonatal, babesiose e anemias hemolíticas de causa indefinida) foram pouco prevalentes e ocorreram principalmente em eqüinos com menos de um mês de idade $(p<0,005)$. Testes sorológicos e de imunofluorescência para Leptospira spp. foram realizados nos casos de anemia hemolítica sem causa definida, porém, os resultados foram negativos.

As afecções mais prevalentes do sistema cardiovascular foram os infartos intestinais associados à tromboembolismo induzido por larvas de Strongylus vulgaris. Lesões vasculares associadas à migração desse parasita, principalmente na artéria mesentérica e seus ramos, são freqüentes em eqüinos, mas nem sempre são causa de morte. Outras causas de morte como a arterite com trombos e a ruptura de aneurisma na artéria cólica também estavam ou poderiam estar associados à infestação por esse parasita.

Afecções no sistema reprodutor acometeram quase que somente fêmeas e incluíram principalmente endometrites, que representaram $45,4 \%$ dos casos. A osteopetrose relatada no sistema endócrino acometeu eqüinos 
de até cinco meses de idade e estava associada à suplementação excessiva com iodo das éguas prenhes durante a gestação (Silva et al. 1987).

Agradecimentos.- Ao Prof. José Henrique Silva pela orientação na análise estatística.

\section{REFERÊNCIAS}

Baker J.R. \& Ellis C.E. 1981. A survey of post mortem findings in 480 horses 1958 to 1980. I. Causes of death. Eq. Vet. J. 13:43-46.

Barros C.S.L., Barros S.S., Santos M.N. \& Souza M.A. 1984. Leucoencefalomalacia em eqüinos no Rio Grande do Sul. Pesq. Vet. Bras. 4:101-107.

Blikslager A.T., Bowman K.F., Haven M.L., Tate Jr L.P. \& Bristol D.G. 1992. Pedunculated lipomas as a cause of intestinal obstruction in horses: 17 cases (1983-1990). J. Am. Vet. Med. Assoc. 201:12491252.

Cohen N.D. 2002. Factors associated with increased risk of colic, p.101103. In: Mair T., Divers T. \& Ducharme N. (Ed.), Manual of Equine Gastroenterology. W.B. Saunders, London. 540p.

Egenvall A., Penell J.C., Bonnett B.N., Olson P. \& Pringle J. 2005. Morbidity of Swedish horses insured for veterinary care between 1997 and 2000: Variations with age, sex, breed and location. Vet. Rec. 157:436-443.

Fretz P.B., Hamilton G.F., Barber S.M. \& Ferguson J.G. 1983. Management of umbilical hernias in cattle and horses. J. Am. Vet. Med. Assoc. 183:550-552.

Gift L.J., Gaughan E.M., DeBowes R.M., Pintchuk P.A., Nickels F.A. \& Foreman J.H. 1993. Jejunal intussusception in adult horses: 11 cases (1981-1991). J. Am. Vet. Med. Assoc. 202:110-112.

Godoy R.A. \& Neto A.R.T. 2007. Cólica em eqüinos, p.571-623 In: RietCorrea F., Schild A.L., Lemos R.A.A. \& Borges J.R.J. (Ed.), Doenças de Ruminantes e Eqüinos. $3^{a}$ ed. Palotti, Santa Maria, RS. 694p.

Hackett R.P. 2002. Displacement of the large colon, p.284-287 In: Mair T., Divers T. \& Ducharme N. (Org.), Manual of Equine Gastroenterology. W.B. Saunders, London. 566p.

Martin S. 1987. Veterinary Epidemiology: Principles and methods. lowa State University Press, Ames. 343p.
Mee A.M., Cripps P.J. \& Jones R.S. 1998. A retrospective study of mortality associated with general anaesthesia in horses: Emergency procedures. Vet. Rec. 142:307-309.

Pimentel L.A., Oliveira D.M., Neves P.B., Dantas A.F.M. \& Riet-Correa F. 2007. Doenças do sistema nervoso central de eqüídeos no semiárido. 13ํㅡㄹ Enapave, Campo Grande, MS, p.147-148. (Resumo)

Reichmann P., Dearo A.C.O. \& Rodrigues T.C. 2008. Ocorrência de doenças oftalmológicas em eqüinos utilizados para tração urbana na cidade de Londrina, PR. Ciência Rural, 38(9):2525-2528.

Riet-Correa F., Meirelles A.M., Barros C.S.L. \& Gava A. 1998. Equine leukoencephalomalacia in Brazil, p.479-482. In: Garland T. \& Barr A.V. (Ed.), Toxic Plants and other Natural Toxicants. CAB International, Wallingford. 585p.

Rodrigues A., Fighera R.A., Souza T.A., Schild A.L., Soares M.P., Milano J. \& Barros C.S.L. 2005. Surtos de tripanossomíase por Trypanosoma evansi em eqüinos no Rio Grande do Sul: aspectos epidemiológicos, clínicos, hematológicos e patológicos. Pesq. Vet. Bras. 25:239-249.

Rodrigues A., Fighera R.A., Souza T.M., Schild A.L. \& Barros C.S.L. 2009. Neuropathology of naturally occurring Trypanosoma evansi infection of horses. Vet. Pathol. 46:251-258.

Rooney J.R. \& Robertson J.L. 1996. Gastrointestinal tract and adnexa, p.57-114 In: Rooney J.R. \& Robertson J.L. (Ed.), Equine Pathology. lowa State University Press, Ames. 482p.

Sallis E.S.V., Pereira D.I.B. \& Raffi M.B. 2003. Pitiose cutânea em eqüinos: 14 casos. Ciência Rural 33:899-903.

SAS 1996. Analyses System. SAS Institute, Cary. 705p.

Schumacher J. 2002. Diseases of the small colon and rectum, p.299316. In: Mair T., Divers T. \& Ducharme N. (Ed.), Manual of Equine Gastroenterology. W.B. Saunders, London. 540p.

Silva C.A.M., Merkt H., Bergamo P.N.L., Barros S.S., Barros C.S.L., Santos M., Hoppen H.O., Heidemann P. \& Meyer H. 1987. Consequences of excess iodine supply in a Thoroughbred stud in southern Brazil. J. Reprod. Fertil. 35(Suppl.):529-533

Trotte M.N.S., Santos I.B., Miranda L.H.M., Amorim A.R., Borges J.R.J. \& Menezes R.C. 2008. Histopatologia de lesões tumoriformes presentes na cavidade nasal de eqüídeos do Brasil. Ciência Rural, 38(9):2540-2544.

Valentine B.A. 2006. Survey of equine cutaneous neoplasia in the Pacific Northwest. J. Vet. Diag. Invest. 18:123-126 